\title{
Exploring the Relationship between Obsessive-compulsive Characteristics and Symptoms of Eating Disorders in a Nonclinical Population
}

\author{
John Paulson ${ }^{1, *}$ \\ ${ }^{1}$ Social Work Department, University of Southern Indiana, USA \\ *Correspondence: Social Work Department, University of Southern Indiana, 8600 University Boulevard, Evansville, \\ IN 47712, USA. Tel: 1-812-465-7103. E-mail: ajpaulson@usi.edu
}

Received: December 4, 2019 Accepted: January 3, 2020 Online Published: January 19, 2020

doi:10.5430/wjss.v7n1p25 URL: https://doi.org/10.5430/wjss.v7n1p25

\begin{abstract}
Previous research has documented similarities between symptoms of Obsessive-Compulsive Disorder (OCD), Anorexia Nervosa, and Bulimia Nervosa and elevated comorbidity between these conditions in clinical samples, with the relationship between OCD and Anorexia being stronger than between OCD and Bulimia. Researchers adopting a continuum view of psychopathology have also found that individuals with sub-clinical expressions of obsessive-compulsive symptoms resemble their clinical counterparts in several ways. The goal of the current study was to explore whether or not the observed relationship between obsessive-compulsive symptoms and eating disorder symptoms observed in clinical populations would also be observed in a nonclinical population. 264 participants from a college sample completed self-report measures of these symptoms. A positive correlation was found between scores on obsessive-compulsive, anorexia and bulimia instruments, and reflective of their clinical counterparts the relationship between obsessive-compulsive and anorexia symptoms was more significant than the one between obsessive compulsive symptoms and bulimia symptoms. Implications and limitations for research and clinical practice are discussed.
\end{abstract}

Keywords: nonclinical obsessives, eating disorder symptoms, continuum view of psychopathology

\section{Introduction}

\subsection{The Obsessive-Compulsive Spectrum}

Previous research has documented an elevated rate of comorbidity between the clinical diagnoses of Obsessive-Compulsive Disorder (OCD) and eating disorders, specifically Anorexia and Bulimia (Pigott et al., 1991; Thiel, Broocks, Ohlmeier, Jacoby, \& Schüssler, 1995). Trends in these studies also suggest that OCD and obsessive symptoms appear to be more strongly associated with Anorexia than with Bulimia and that the comorbid presence of OCD among individuals with primary eating disorders is more common than the presence of an eating disorder in someone with primary OCD (Bang et. al., 2020; Jarry \& Vaccarino, 1996; Levinson et. al., 2019). The presence of increased eating disorder symptoms or a co-morbid eating disorder often complicates treatment and leads to poorer prognosis and outcomes, more so with anorexia symptoms than bulimia symptoms (Sallet et. al., 2010; Simpson et. al., 2013). Investigators examining these similarities have suggested that OCD, Anorexia and Bulimia may belong to a common spectrum of disorders (Hollander \& Benzaquen, 1997; Hollander \& Wong, 1995).

The obsessive-compulsive spectrum disorders constitute a variety of disorders from different diagnostic classifications, including impulse control and eating disorders. Disorders in the Obsessive-Compulsive spectrum seem to share common neuroanatomical and neurochemical characteristics with OCD, including the role of the prefrontal cortex, basal ganglia, thalamus and the neurotransmitter serotonin in each disorder. The conditions also share phenomenological similarities in the experience of recurrent, unwanted, intrusive thoughts and images that carry some degree of threat valence and subsequent repetitive, ritualistic, compulsive behavior aimed at addressing or neutralizing perceived threats (Hollander \& Benzaquen, 1997; Hollander \& Wong, 1995). 
The accumulation of research supporting an Obsessive-Compulsive Spectrum led to changes in DSM-5. OCD was removed from the anxiety disorder category and a new section titled the Obsessive-Compulsive and Related Disorders was created. While not all the conditions theorists consider as being a part of the obsessive-compulsive spectrum were included in this new category (including Anorexia and Bulimia, still categorized in the Feeding and Eating Disorders section), this new category does include the conditions previously included in the impulse control disorder section of trichotillomania and excoriation and also includes Body Dysmorphic Disorder, which was previously categorized with the eating disorders (APA, 2013).

\subsection{Nonclinical Obsessives}

The continuum view of psychopathology varies from the more traditional medical, categorical, dichotomous model that emphasizes distinct categories where one either has or does not have a condition. Instead it emphasizes that not only do diagnoses exist along a continuum of severity (from mild to severe presentations of a condition) but that their symptoms and traits do as well. This extends into nonclinical populations, suggesting that individuals can have aspects or characteristics of a condition without having the diagnosis (Widiger, 1992). This emphasis on continuum, developmental and spectrum conceptualizations of psychiatric disorders is now reflected in the latest edition of the Diagnostic and Statistical Manual of Mental Disorders Fifth Edition ( $5^{\text {th }}$ ed.; DSM-5; American Psychiatric Association [APA], 2013).

Investigators supporting a continuum view of psychopathology have studied the expression of psychiatric symptoms and characteristics in nonclinical samples. Such individuals are ones that either do not meet the criteria for a disorder or who are sampled from populations other than psychiatric treatment settings. A sizeable amount of research has been conducted on what researchers refer to as nonclinical obsessives (for a comprehensive review of this literature see Gibbs, 1996). This research shows that nonclinical obsessives share several similar characteristics to those with OCD, just to a lesser degree than those with the disorder. Similar to those diagnosed with OCD nonclinical obsessives commonly report obsessions with themes that focus on contamination, harm and doubt. They also engage in similar compulsive behaviors of repetitive checking, cleaning, and hoarding of items. Consistent with patterns of comorbidity between OCD, anxiety, Post-Traumatic Stress Disorder and mood disorders in clinical populations, nonclinical obsessives exhibit increased disturbances in general mood, depression, anxiety and post-traumatic symptoms (Gibbs, 1996; Huppert et. al., 2005). Individuals with OCD have been shown to display cognitive deficits in short-term memory, nonverbal memory, and set shifting, and these same deficits have also been observed in nonclinical obsessives (Frost, Lahart, Dugas, \& Sher, 1988; Gibbs, 1996; Zohar, LaBuda, \& Moschel-Ravid, 1995).

While significant research has demonstrated similarities between nonclinical obsessives and those with OCD, there has been minimal investigation into whether the relationship between OCD and eating disorders is also observed in nonclinical populations. Frost, Sher and Geen (1986) examined the relationship between obsessive-compulsive symptoms and eating restraint (dieting, binging, affective state during eating) in a sample of undergraduate females. They found that those scoring higher in obsessive-compulsive symptoms had higher scores on the eating restraint scale, had more episodes of binge eating, and had higher rates of eating associated with negative affect. Davis, Claridge and Cerullo (1997) explored the relationship between personality characteristics and Bulimia in a non-clinical sample of female college students. These investigators actually found a negative relationship between obsessive-compulsive characteristics and symptoms of Bulimia. They speculate that this finding was due to the more significant relationship between obsessive-compulsive symptoms and anorexia symptoms than with bulimia symptoms. In perhaps the largest study on the topic, Formea and Burns (1995) examined the relationship between obsessive-compulsive symptoms and bulimia symptoms in a group of 1703 college students. They found a positive correlation between obsessive-compulsive and bulimia symptoms.

Consistent with this line of investigation, the current study set out to briefly examine the relationship between obsessive-compulsive characteristics and symptoms of eating disorders in a nonclinical population. The purpose of this study was to explore whether such a positive relationship would be observed in a nonclinical population, and if so whether that relationship would resemble trends seen in clinical populations of obsessive-compulsive symptoms being more strongly related with anorexia symptoms than with bulimia symptoms.

\section{Methods}

\subsection{Participants}

264 participants, consisting of 80 males and 184 females, from a large Midwestern university completed the study. The participants ranged in age from 18 to $53(M=20.57)$. Ethnic identification in the sample consisted of $91.2 \%$ 
Caucasian, 5.7\% African American, $0.8 \%$ Asian, and $0.8 \%$ Chicano. $1.5 \%$ of participants in the study identified their ethnic identity as "other". All participants in the study were volunteers at least eighteen years of age, were currently enrolled in either an introduction to psychology course or introduction to social work course and had not completed a research methods course. Some participants received extra course credit for participating in the study.

\subsection{Materials}

Maudsley Obsessional-Compulsive Inventory (MOCI)-The Maudsley Obsessional-Compulsive Inventory (Hodgson \& Rachman, 1977) is a thirty-item self-report measure of obsessive-compulsive symptoms. Questions are posed in a dichotomous, yes/no format. The scale contains four subscales related to different general categories of obsessive-compulsive symptoms (checking, cleaning, slowness, and doubting). A composite score for the MOCI, as well as scores for each subscale, are obtained by summing the number of responses that positively relate to obsessive-compulsive concerns. The MOCI has demonstrated good internal consistency, test-retest reliability and validity (Hodgson \& Rachman, 1977; Sternberger \& Burns, 1990a).

Eating Attitudes Test (EAT)-The Eating Attitudes Test (Garner \& Garfinkel, 1979) is a forty-item self-report instrument that measures symptoms of anorexia nervosa. Test items are set up in a six-point Likert format. Higher scores on the instrument represent the increased presence of anorexia symptoms. The authors of the test recommend a clinical cutoff score of thirty, with scores above this being suggestive and supportive of a potential diagnosis of Anorexia. The EAT has demonstrated good internal consistency, discriminate validity and responsiveness to improvements from treatment (Garner \& Garfinkel, 1979; Mintz \& O’Halloran, 2000).

The Bulimia Test-Revised (BULIT-R)-The Bulimia Test-Revised (Thelen, Farmer, Wonderlich, \& Smith, 1991) is a self-report instrument that measures symptoms of bulimia nervosa. The test includes twenty-eight items set in a Likert format with higher scores on the instrument reflecting the greater presence of bulimia symptoms. Scores of 104 and above on the instrument suggest a diagnosis of bulimia nervosa. In an effort to reduce the possibility of false negatives, Fischer and Corcoran (1994) recommend a cutting score of 85. The BULIT-R has demonstrated good internal consistency, test-retest reliability, as well as concurrent, construct and discriminate validity (Thelen, Farmer, Wonderlich, \& Smith, 1991; Thelen, Mintz, \& Vander Wal, 1996; Welch, Thompson, \& Hall, 1993).

\subsection{Procedure}

After receiving IRB approval and obtaining informed consent from each participant, the primary investigator administered the test battery to participants during a regularly scheduled meeting of their class. The test battery consisted of the MOCI, EAT, and BULIT-R. Total scores for each scale, as well as subscale scores for the MOCI, were then calculated.

\section{Results}

Descriptive statistics, including means, standard deviations, and score ranges are presented in Table 1.

Table 1. Means, Standard Deviations, Minimum and Maximum Scores on All Measures

\begin{tabular}{lllll}
\hline Variables & Mean & SD & Minimum & Maximum \\
\hline MOCI & 10.05 & 4.68 & 2.00 & 26.00 \\
EAT & 14.82 & 9.56 & 2.00 & 58.00 \\
BULIT-R & 50.53 & 18.27 & 14.00 & 115.00
\end{tabular}

Note: $\mathrm{N}=264$. MOCI=Maudsley Obsessional-Compulsive Inventory; EAT-Eating Attitudes Test; BULIT-R=Bulimia Test-Revised

One-tailed correlation coefficients were then calculated for the total scores on the MOCI, the MOCI subscales, the EAT and the BULIT-R. 
Table 2. Correlations between MOCI, EAT, and BULIT-R Scores

\begin{tabular}{llllllll}
\hline Measure & 1 & 2 & 3 & 4 & 5 & 6 & 7 \\
\hline 1. MOCIT & & & & & & \\
2. CHECK & $.781^{* *}$ & & & & & \\
3. CLEAN & $.764^{* *}$ & $.407^{* *}$ & & & & \\
4. SLOW & $.523^{* *}$ & $.165^{* *}$ & $.384^{* *}$ & & & \\
5. DOUBT & $.625^{* *}$ & $.468^{* *}$ & $.144^{* *}$ & $.125^{*}$ & & \\
6. EAT & $.303^{* *}$ & $.177^{* *}$ & $.266^{* *}$ & $.236^{* *}$ & $.158^{* *}$ & \\
7. BULIT & $.230^{* *}$ & $.126^{*}$ & $.150^{* *}$ & $.174^{* *}$ & $.200^{* *}$ & $.639^{* *}$ & \\
\hline
\end{tabular}

Note: $\mathrm{N}=264$. MOCIT $=$ MOCI Total; $\mathrm{CHECK}=$ checking subscale; CLEAN=cleaning subscale; $\mathrm{SLOW}=$ slowness subscale; DOUBT=doubting subscale; EAT=EAT total; BULIT=BULIT-R total

${ }^{*} \mathrm{p}<.05 .{ }^{* *} \mathrm{p}<.01$.

Statistically significant correlations were observed between all variables measured. Total scores on the MOCI and total scores on both the EAT $(r=.303, p=.000)$ and BULIT-R $(r=.230, p=.000)$ were positively correlated, with the correlation between the MOCI and the EAT being higher than that between the MOCI and the BULIT-R. All four subscales of the MOCI were significantly correlated with the EAT and BULIT-R total scores as well. These correlations, while significant, are minimal and suggest that the total MOCI score as a global rating of obsessive and compulsive symptoms has a stronger relationship to eating disorder symptoms than any particular subtype of obsessive-compulsive symptoms measured by the MOCI.

\section{Discussion}

This investigation set out to explore the relationship between obsessive-compulsive, anorexia, and bulimia-related symptoms in a nonclinical sample. Although there is a well-established connection between Obsessive-Compulsive Disorder, Anorexia, and Bulimia in clinical populations, very few studies have examined this trend in nonclinical populations. Those that have explored this dynamic have almost exclusively explored the connection between obsessive-compulsive and bulimia symptoms, as opposed to examining any connection to anorexia symptoms.

The primary hypotheses of this investigation, that scores on self-report measures of obsessive-compulsive, anorexia, and bulimia symptoms would be positively correlated in a nonclinical sample, and that the relationship between obsessive-compulsive and anorexia symptoms would be higher than that between obsessive-compulsive and bulimia symptoms, were both supported. These finding coincide with trends in the clinical literature (Pigott et al., 1991; Thiel, Broocks, Ohlmeier, Jacoby, \& Schüssler, 1995) generally, and specifically with observations detailing higher rates between OCD and Anorexia than Bulimia (Bang et. al., 2020; Jarry \& Vaccarino, 1996; Levinson et. al., 2019). These findings are also comparable to the previous literature on eating disorder symptoms in samples of non-clinical obsessives (Davis, Claridge, \& Cerullo, 1997; Formea \& Burns, 1995; Frost, Sher, \& Geen, 1986).

Results from this study showed that the non-clinical obsessive sample under current investigation performed very similarly to clinical counterparts with regards to relationships between these symptoms and those of symptoms of eating disorders. These findings add continued support to the continuum view of psychopathology (Widiger, 1992) and to reported similarities between non-clinical obsessives and those with Obsessive-Compulsive Disorder (Formea \& Burns, 1995; Gibbs, 1996; Huppert et al., 2005; Zohar, LaBuda, \& Moschel-Ravid, 1995). The current study is one of the only that has explored and documented this trend of a positive relationship not only between obsessive-compulsive symptoms and eating disorder symptoms, but a stronger relationship between obsessive-compulsive and anorexia symptoms than bulimia symptoms in a nonclinical population.

The current investigation does have several notable limitations. The scope of this pilot study was limited, and the sample size was relatively small. The correlations observed between variables, although significant, are also rather small. One explanation of these small values is the use of a nonclinical sample, which tend to perform similarly to, yet more moderately than, clinical samples. Another possible explanation for the modest correlations could also be the instruments used to assess these symptoms. Although the MOCI is perhaps the most widely used instrument in research on nonclinical obsessives (Sternberger \& Burns, 1990a), previous studies using this instrument to assess obsessive-compulsive symptoms in individuals with eating disorders have produced mixed findings (Fahy, 1991; 
Weiss \& Ebert, 1983). While the EAT and the BULIT-R have been successfully used with a number of nonclinical samples (Formea \& Burns, 1995; Wilson \& Mercer, 1990), it is also important to remember that they are instruments meant for clinical populations and may not fully identify eating disturbances in the general population. Mintz and O'Halloran (2000), for example, indicate that the EAT may have a high false-positive rate and weak predictive capabilities when used in nonclinical samples. Future investigation could consider the benefit of utilizing different measures for these variables.

\section{Conclusion}

Despite these limitations, the current study does provide support for the relationship between obsessive-compulsive, anorexia, and bulimia symptoms observed in both clinical and non-clinical populations. It also bolsters observed similarities in the research literature between nonclinical obsessives and clinical populations with OCD generally, and specifically with symptoms of eating disturbance in nonclinical obsessives. In addition to expanding and enriching the literature in this area, the current investigation also has potential beneficial clinical implications. Adopting a continuum view of psychopathology reminds clinicians that individuals can possess characteristics and difficulties of conditions, including clinically relevant ones, even if they do not meet the full criteria for a diagnosis. Recognizing patterns of comorbidity between obsessive-compulsive and eating disorders symptoms and diagnoses, including possible subclinical presentations, and that the combined presence of each can complicate treatment could assist providers in developing and improving assessment and intervention strategies. While the literature on NCO's has not received much recent attention, research on NCO's and other nonclinical populations further supports developmental and continuum views of psychopathology, both of which offer continued research and clinical utility.

\section{References}

American Psychiatric Association. (2013). Diagnostic and statistical manual of mental disorders (5th ed.). Washington, DC: Author. https://doi.org/10.1176/appi.books.9780890425596

Bang, L., Kristensen, U. B., Wisting, L., Stedal, K., Garte, M., Minde, Å., \& Rø, Ø. (2020). Presence of eating disorder symptoms in patients with obsessive-compulsive disorder. BMC Psychiatry, 20(1), 1-10. https://doi.org/10.1186/s12888-020-2457-0

Davis, C., Claridge, G., \& Cerullo, D. (1997). Personality factors and weight preoccupation: A continuum approach to the association between eating disorders and personality disorders. Journal of Psychiatric Research, 31(4), 467-480. https://doi.org/10.1016/S0022-3956(97)00006-X

Fahy, T. A. (1991). Obsessive-compulsive symptoms in eating disorders. Behaviour Research and Therapy, 29(2), 113-116. https://doi.org/10.1016/0005-7967(91)90038-5

Fischer, J., \& Corcoran, K. (1994). Measures for Clinical Practice: A Sourcebook (2nd Ed.). New York: The Free Press.

Formea, G. M., \& Burns, G. L. (1995). Relation between the syndromes of bulimia nervosa and obsessive compulsive disorder. Journal of Psychopathology and Behavioral Assessment, 17(2), 167-176. https://doi.org/10.1007/BF02229016

Frost, R. O., Lahart, C. M., Dugas, K. M., \& Sher, K. J. (1988). Information processing among non-clinical compulsives. Behaviour Research and Therapy, 26(3), 275-277. https://doi.org/10.1016/0005-7967(88)90011-3

Frost, R. O., Sher, K. J., \& Geen, T. (1986). Psychopathology and personality characteristics of nonclinical compulsive checkers. Behaviour Research and Therapy, 24(2), 133-143. https://doi.org/10.1016/0005-7967(86)90084-7

Garner, D. M., \& Garfinkel, P. E. (1979). The Eating Attitudes Test: An index of the symptoms of anorexia nervosa. Psychological Medicine, 9(2), 273-279. https://doi.org/10.1017/S0033291700030762

Gibbs, N. A. (1996). Nonclinical populations in research on obsessive-compulsive disorder: A critical review. Clinical Psychology Review, 16(8), 729-773. https://doi.org/10.1016/S0272-7358(96)00043-8

Hodgson, R. J., \& Rachman, S. (1977). Obsessional-compulsive complaints. Behaviour Research and Therapy, 15(5), 389-395. https://doi.org/10.1016/0005-7967(77)90042-0

Hollander, E., \& Benzaquen, S. D. (1997). The obsessive-compulsive spectrum disorders. International Review of Psychiatry, 9(1), 99-110. https://doi.org/10.1080/09540269775628 
Huppert, J. D., Moser, J. S., Gershuny, B. S., Riggs, D. S., Spokas, M., Filip, J., Hajcak, G., Parker, H.A., Baer, L., \& Foa, E. B. (2005). The relationship between obsessive-compulsive and posttraumatic stress symptoms in clinical and non-clinical samples. Journal of Anxiety Disorders, 19(1), 127-136. https://doi.org/10.1016/j.janxdis.2004.01.001

Hollander, E., \& Wong, C. M. (1995). Obsessive-compulsive spectrum disorders. The Journal of Clinical Psychiatry, 56(Suppl 4), 3-6.

Jarry, J. L., \& Vaccarino, F. J. (1996). Eating disorder and obsessive-compulsive disorder: Neurochemical and phenomenological commonalities. Journal of Psychiatry \& Neuroscience, 21(1), 36-48.

Levinson, C. A., Brosof, L. C., Ram, S. S., Pruitt, A., Russell, S., \& Lenze, E. J. (2019). Obsessions are strongly related to eating disorder symptoms in anorexia nervosa and atypical anorexia nervosa. Eating Behaviors, 34, 101298. https://doi.org/10.1016/j.eatbeh.2019.05.001

Mintz, L. B., \& O'Halloran, M. S. (2000). The Eating Attitudes Test: Validation with DSM--IV Eating Disorder Criteria. Journal of Personality Assessment, 74(3), 489-503. https://doi.org/10.1207/S15327752JPA7403_11

Pigott, T. A., Altemus, M., Rubenstein, C. S., Hill, J. L., Bihari, K., L’Heureux, F., Bernstein, S., \& Murphy, D. L. (1991). Symptoms of eating disorders in patients with obsessive-compulsive disorder. The American Journal of Psychiatry, 148(11), 1552-1557. https://doi.org/10.1176/ajp.148.11.1552

Sallet, P. C., de Alvarenga, P. G., Ferrão, Y., de Mathis, M. A., Torres, A. R., Marques, A., Hounie, A. G., Fossaluza, V., do Rosario, M. C., Fontenelle, L. F., Petribu, K., \& Fleitlich-Bilyk, B. (2010). Eating disorders in patients with obsessive-compulsive disorder: prevalence and clinical correlates. The International Journal of Eating Disorders, 43(4), 315-325. https://doi.org/10.1002/eat.20697

Simpson, H. B., Wetterneck, C. T., Cahill, S. P., Steinglass, J. E., Franklin, M. E., Leonard, R. C., Weltzin, T. E., \& Riemann, B. C. (2013). Treatment of obsessive-compulsive disorder complicated by comorbid eating disorders. Cognitive Behaviour Therapy, 42(1), 64-76. https://doi.org/10.1080/16506073.2012.751124

Sternberger, L. G., \& Burns, G. L. (1990a). Compulsive Activity Checklist and the Maudsley Obsessional-Compulsive Inventory: Psychometric properties of two measures of obsessive-compulsive disorder. Behavior Therapy, 21(1), 117-127. https://doi.org/10.1016/S0005-7894(05)80193-5

Thelen, M. H., Farmer, J., Wonderlich, S., \& Smith, M. (1991). A revision of the Bulimia Test: The BULIT-R. Psychological Assessment: A Journal of Consulting and Clinical Psychology, 3(1), 119-124. https://doi.org/10.1037/1040-3590.3.1.119

Thelen, M. H., Mintz, L. B., \& Vander Wal, J. S. (1996). The Bulimia Test-Revised: Validation with DSM-IV criteria for bulimia nervosa. Psychological Assessment, 8(2), 219-221. https://doi.org/10.1037/1040-3590.8.2.219

Thiel, A., Broocks, A., Ohlmeier, M., Jacoby, G. E., \& Schüssler, G. (1995). Obsessive-compulsive disorder among patients with anorexia nervosa and bulimia nervosa. The American Journal of Psychiatry, 152(1), 72-75. https://doi.org/10.1176/ajp.152.1.72

Weiss, S. W., \& Ebert, M. H. (1983). Psychological and behavioral characteristics of normal-weight bulimics and normal-weight controls. Psychosomatic Medicine, 45(4), 293-303. https://doi.org/10.1097/00006842-198308000-00004

Welch, G., Thompson, L., \& Hall, A. (1993). The BULIT-R: Its Reliability and Clinical Validity as a Screening Tool for DSM-III-R Bulimia Nervosa in a Female Tertiary Education Population. International Journal of Eating Disorders, 14(1), 95-105. https://doi.org/10.1002/1098-108X(199307)14:1<95::AID-EAT2260140113>3.0.CO;2-Z

Widiger, T. A. (1992). Categorical versus dimensional classification: Implications from and for research. Journal of Personality Disorders, 6(4), 287-300. https://doi.org/10.1521/pedi.1992.6.4.287

Wilson, J. F., \& Mercer, J. C. (1990). An electrophysiological correlate of Eating Attitudes Test scores in female college students. Psychological Medicine, 20(4), 973-975. https://doi.org/10.1017/S0033291700036679

Zohar, A. H., LaBuda, M., \& Moschel-Ravid, O. (1995). Obsessive-compulsive behaviors and cognitive functioning: A study of compulsivity, frame shifting and type an activity patterns in a normal population. Neuropsychiatry, Neuropsychology, \& Behavioral Neurology, 8(3), 163-167. 